\title{
Angle and Polarization Diversity in Compact Dual-Antenna Terminals with Chassis Excitation
}

\author{
Hui Lit ${ }^{1,2}, \underline{\text { Buon Kiong Lau }}{ }^{1}$, and Sailing $\mathrm{He}^{2}$ \\ ${ }^{1}$ Department of Electrical and Information Technology, Lund University, Box 118, SE-221 00 Lund, Sweden \\ E-mail: \{Hui.Li, Buon-Kiong.Lau\}@eit.lth.se \\ ${ }^{2}$ School of Electrical Engineering, Royal Institute of Technology, S-100 44 Stockholm, Sweden \\ E-mail: sailing@kth.se
}

\begin{abstract}
For multiple antennas on a compact terminal chassis, chassis current excitation can severely increase mutual coupling between the antennas. This is because the same characteristic mode of the chassis may be strongly excited by more than one antenna. Here, we propose to use one antenna that exploits chassis excitation and introduce a second antenna that minimizes chassis excitation. In this way, angle and polarization diversity can be realized. The concept is illustrated and verified through the design of a practical dual-antenna prototype at $920 \mathrm{MHz}$, which achieves an isolation of over $30 \mathrm{~dB}$ and total efficiencies of above $80 \%$.
\end{abstract}

\section{Introduction}

Implementing multiple antennas in both base stations and user terminals is a key solution for increasing channel capacity without sacrificing additional frequency spectrum and transmit power [1]. In reality, a user terminal is typically compact (e.g., a mobile phone), which requires the multiple antennas to be closely spaced. It is known that closely spaced antennas suffer from strong mutual coupling, which in turn causes degradation in the expected overall antenna system performances, including bandwidth, efficiency and channel capacity [1].

Most of the existing decoupling techniques suitable for mobile terminals focus on relatively high frequency bands, such as the WLAN, DCS1800 and UMTS bands [1-3]. This is despite that the bigger challenge is in decoupling terminal antennas for frequency bands below $1 \mathrm{GHz}$, where the isolation is typically lower than $6 \mathrm{~dB}$. The poor isolation is mainly because the chassis can play an important role in determining the mutual coupling between the antennas [4]. For low frequency bands, the chassis can act not only as a common ground plane, but also as a radiator shared by more than one antenna. Thus, the radiation patterns can be severely modified by the chassis, such that they are less orthogonal to one another in both angular and polarization properties. In [4], the mutual coupling is reduced for a dual-antenna prototype by optimizing the position of one antenna, with the second antenna located at one short edge of the $100 \mathrm{~mm} \times 40 \mathrm{~mm}$ chassis. It is found that coupling is minimized when the first antenna is located at the center of the chassis, which corresponds to the position of least chassis excitation for that antenna. However, in some cases, this solution of locating one antenna at the center of the chassis may not be practical.

In this paper, a novel and practical concept is proposed to reduce mutual coupling between multiple antennas on a mobile chassis in the presence of chassis excitation. The idea is to use only one antenna to exploit chassis excitation, whereas the second one (or the other ones, if more than two antennas) is designed to avoid chassis excitation. To illustrate and verify the concept, an example dual-antenna prototype working at $920 \mathrm{MHz}$ is designed and implemented. A typical folded monopole is used to take advantage of the chassis, whereas a coupled-fed, capacitively-loaded loop (CLL) is employed to prevent chassis excitation. The proposed CLL provides similar radiation properties as a small loop (i.e., a magnetic dipole), but unlike a small loop, it is well matched to $50 \Omega$ and can be easily printed onto the chassis. Based on this setup, angle and polarization diversity is effectively realized in the antenna radiation patterns. Moreover, isolation of over $30 \mathrm{~dB}$ and efficiencies of above $80 \%$ are achieved.

\section{Characteristic Mode Analysis}

The chassis, corresponding to that of a typical candybar-type mobile phone, is modeled by a perfectly conducting board of dimensions $100 \mathrm{~mm} \times 40 \mathrm{~mm}$. The intrinsic radiation properties of the chassis are evaluated based on the theory of characteristic mode introduced by Harrington [5]. The first characteristic value (resonant frequency) of the chassis is found to be $1.35 \mathrm{GHz}$ [4], which is near the $900 \mathrm{MHz}$ band for mobile communications. The normalized characteristic electric and magnetic fields on a plane $5 \mathrm{~mm}$ above the chassis at $1.35 \mathrm{GHz}$ are shown in Figure 1 . 


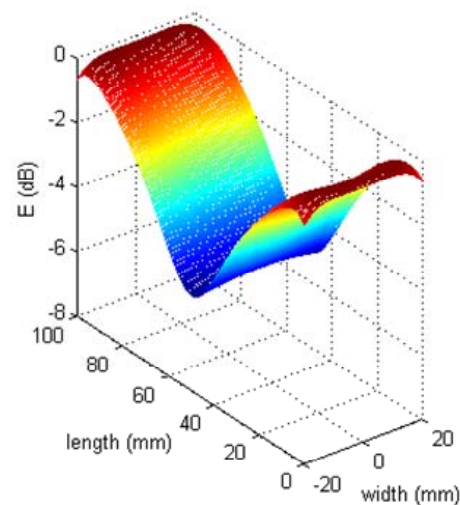

(a)

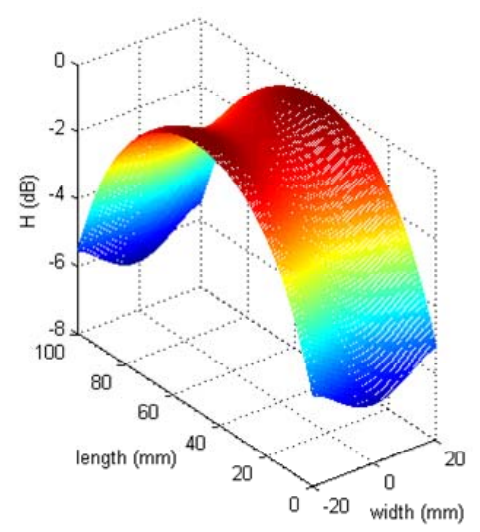

(b)

Figure 1. The normalized magnitude of (a) total electric field and (b) total magnetic field for the characteristic mode 5 $\mathrm{mm}$ above the chassis surface at $1.35 \mathrm{GHz}$.

Figure 1(a) reveals that if two antennas that are responsive to electric field are implemented on the two short edges of the chassis, they both strongly excite the chassis and use it as a radiator. Consequently, the shared radiator leads to severe mutual coupling. As it turns out, most of the antenna types in today's mobile phones, such as monopoles, slot antennas and PIFAs, are electric-field-responsive, and the antennas are often placed at the short chassis edges. Therefore, this phenomenon of shared chassis excitation is largely responsible for the typically poor isolation between terminal antennas observed at frequency bands below $1 \mathrm{GHz}$, even if the electrical distance between them may suggest better isolation [1]. On the contrary, from Figure 1(b), it can be concluded that if the two antennas are responsive to magnetic field, they cannot effectively excite the chassis when they are placed at the two short edges. Good isolation is thus expected. However, the bandwidth of an electrically compact antenna is narrow without the help of the chassis. To achieve a good compromise between the isolation and the bandwidth, an electric-field-responsive antenna is used to exploit chassis excitation to achieve wider bandwidth, whereas a magnetic-field-responsive antenna prevents chassis excitation and enhances isolation. In this way, angle and polarization diversity can be effectively achieved for the two antennas.

\section{Antenna System with Enhanced Isolation}

\subsection{Antenna Simulations}

In this section, based on the concept presented above, a practical dual-antenna prototype consisting of a folded monopole (i.e., a commonly used antenna type in mobile phones) and a printed CLL is proposed. The geometries of the antenna system are presented in Figure 2(a). Both antennas are printed on a substrate with the permittivity of 2.45, loss tangent of 0.003 and thickness of $0.8 \mathrm{~mm}$. The conductivity of the copper layer is $5.8 \times 10^{7} \mathrm{~S} / \mathrm{m}$. The monopole is fed by a microstrip line. Inspired by the structure in [6], the printed CLL in our work consists of two half square rings, with the inner ring acting as the matching feed and the outer ring as the main radiator. The loop is implemented on the same plane as the chassis, and takes advantage of one short edge of the chassis to form a complete square ring. The inter-digital capacitor at the outer ring, with both the arm width and arm separation distance of $0.8 \mathrm{~mm}$, is used to provide a capacitive load for the loop. The resonant frequency of the CLL can be changed by varying the length of the arms $\left(L_{c}\right)$.

The simulated scattering (or S) parameters of the antenna system are shown in Figure 2(b). It is seen that both the monopole and CLL are well matched, and the isolation exceeds $30 \mathrm{~dB}$, which is very high for low frequency bands.

The total efficiencies of the two antennas are given by

$$
\eta_{\mathrm{total}, 1}=\eta_{\mathrm{rad}, 1}\left(1-\left|S_{11}\right|^{2}-\left|S_{21}\right|^{2}\right) \text { and } \eta_{\mathrm{total}, 2}=\eta_{\mathrm{rad}, 2}\left(1-\left|S_{22}\right|^{2}-\left|S_{12}\right|^{2}\right) \text {, }
$$

respectively. Due to the high radiation efficiency $\left(\eta_{\mathrm{rad}, 1}, \eta_{\mathrm{rad}, 2}\right)$, good impedance matching $\left(S_{11}, S_{22}<-15 \mathrm{~dB}\right)$ and low mutual coupling $\left(S_{21}, S_{12}<-30 \mathrm{~dB}\right)$, the simulated total efficiencies for the monopole and the loop are very promising, i.e., $91.56 \%$ and $80 \%$, respectively, at $0.925 \mathrm{GHz}$. 


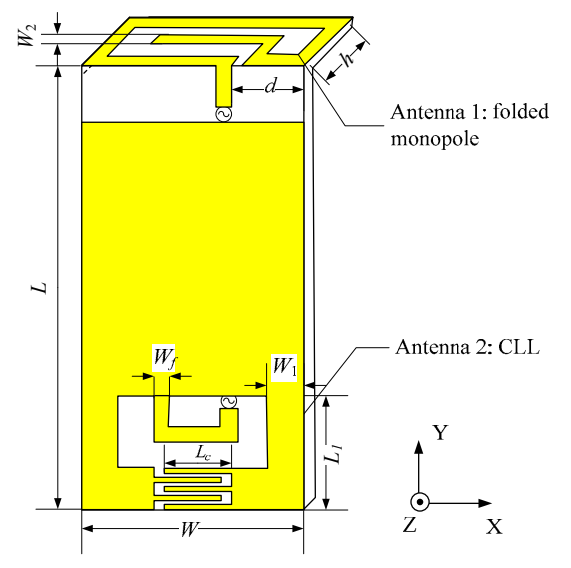

(a)

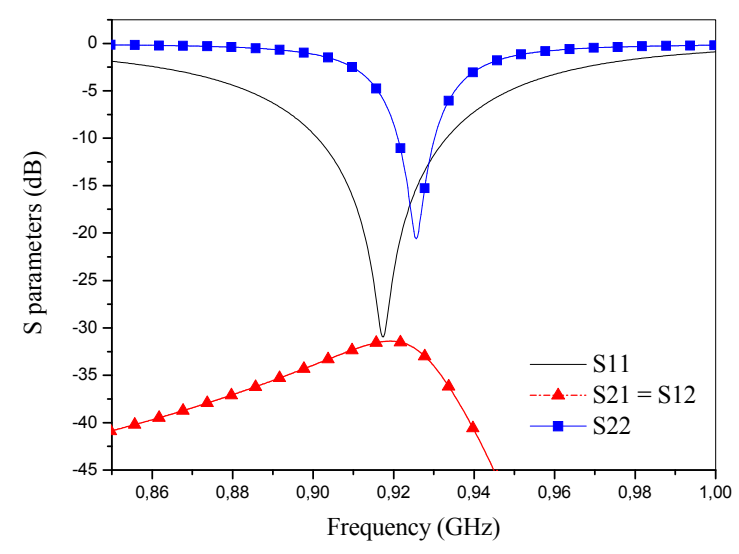

(b)

Figure 2. (a) The geometries of the folded monopole and the magnetic-field-responsive loop. The dimensions are: $L=$ $100 \mathrm{~mm}, W=40 \mathrm{~mm}, L_{1}=15 \mathrm{~mm}, h=6 \mathrm{~mm}, L_{c}=7.5 \mathrm{~mm}, W_{f}=2 \mathrm{~mm}, W_{1}=3 \mathrm{~mm}, W_{2}=1 \mathrm{~mm}, d=12 \mathrm{~mm}$. (b) The simulated $\mathrm{S}$ parameters for the proposed antenna system.

The far field patterns of the proposed antenna system are shown in Figure 3, with the E-theta and E-phi components shown separately. Good polarization diversity is observed. For most of the angles, when the E-phi component is dominant for the monopole antenna, the E-theta component, which is orthogonal to E-phi component, plays the main role for CLL, and vice verse. Angle diversity also contributes to the good isolation between the two antennas. The slight asymmetry observed in the E-phi component of the CLL is attributed to the asymmetric feeding method. The magnitude of the complex correlation coefficient calculated from the far field patterns is 0.003 at $0.925 \mathrm{GHz}$. The effective diversity gain at $1 \%$ probability, for selection diversity combining, is found to be $9.45 \mathrm{~dB}$.

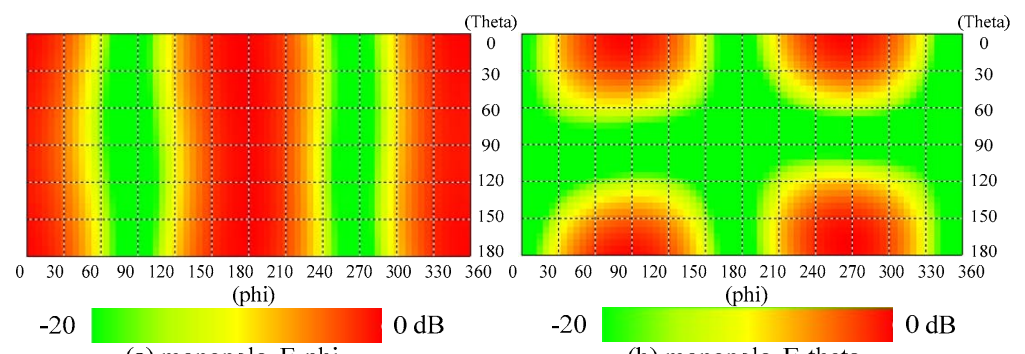

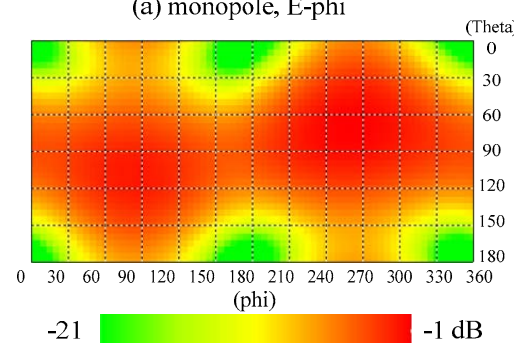

(c) CLL, E-phi
$-20$

(b) monopole, E-theta

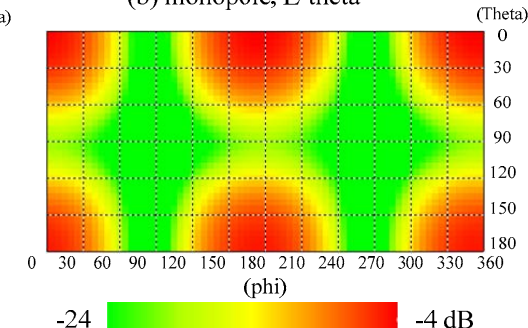

(d) CLL, E-theta

Figure 3. The normalized far field radiation patterns for the monopole and the CLL at $0.925 \mathrm{GHz}$.

\subsection{Antenna Experiments}

The antennas are fabricated as shown in Figure 4(a). The materials used are the same as those in the simulation. The scattering parameters are measured with a vector network analyzer and shown in Figure 4(b). Basically, the measured results agree well with the simulated results. The isolation is a little worse than that in the simulation due to both fabrication tolerance and cable influence. However, isolation of above $20 \mathrm{~dB}$ can still be achieved. The only 

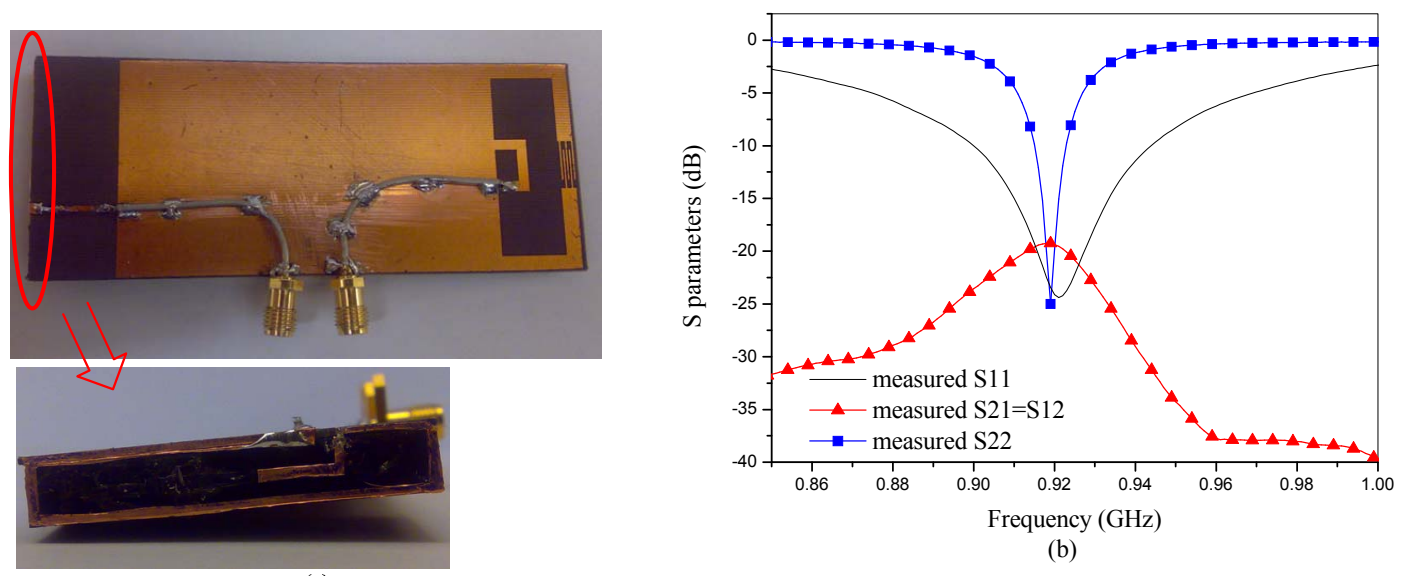

(a)

(b)

Figure 4. The (a) fabricated prototype and (b) measured S parameters of the proposed antenna system.

drawback of the proposed antenna system is the narrow bandwidth $(18 \mathrm{MHz})$ of the CLL. However, this can be compensated through the use of adaptive tuning circuits. Moreover, the CLL can be used as a diversity antenna for only the receive mode, to reduce the bandwidth requirement, while the wider-band monopole is used as the main radiator.

\section{Conclusions}

In this paper, a concept to enhance the isolation between multiple antennas on small terminals is introduced. To exploit chassis excitation, an electric-field-responsive antenna is used as the main radiator. On the other hand, the diversity antenna(s) is designed to be magnetic-field-responsive, in order to avoid chassis excitation. To illustrate and verify the concept, a practical dual-antenna system is proposed. Our results show that angle and polarization diversity is effectively achieved, giving high antenna efficiency $(>80 \%)$, low mutual coupling $(<-30 \mathrm{~dB})$ and high diversity gain $(9.45 \mathrm{~dB})$. Experiments that are performed largely confirm the simulation results.

\section{Acknowledgments}

This work was supported in part by VINNOVA under grant no. 2008-00970 and 2009-04047, and also in part by a scholarship within EU Erasmus Mundus External Cooperation Window Lot 14. The authors are also thankful to Prof. Jørgen Bach Andersen of Aalborg University for helpful discussions.

\section{References}

1. B. K. Lau, "Multiple antenna terminals," in MIMO: From Theory to Implementation, C. Oestges, A. Sibille, and A. Zanella, Eds. San Diego: Academic Press, 2011, pp. 267-298.

2. A. Diallo, C. Luxey, P. L. Thuc, R. Staraj, and G. Kossiavas, "Enhanced two-antenna structures for universal mobile telecommunications system diversity terminals," IET Microw. Antennas Propogat., vol. 2, no. 1, pp. 93-101, 2008.

3. H. Li, J. Xiong, and S. He, "A compact planar MIMO antenna system of four elements with similar radiation characteristics and isolation structure," IEEE Antennas Wireless Propagat. Lett., vol. 8, pp. 1107-1110, 2009.

4. H. Li, B. K. Lau, and Z. Ying, "Optimal multiple antenna design for compact MIMO terminals with ground plane excitation," in Proc. Int. Workshop Antenna Technol. (IWAT), Hong Kong SAR, P. R. China, Mar. 7-9, 2011.

5. R. F. Harrington and J. R. Mautz, "Computation of characteristic modes for conducting bodies," IEEE Trans. Antennas Propagat., vol. AP-19, no. 5, pp. 629-639, Sep. 1971.

6. A. Erentok and R. W. Ziolkowski, "Metamaterial-inspired efficienct electrically small antennas," IEEE Trans. Antennas Propagat., vol. 54, no. 3, pp. 691-706, Mar. 2008. 